\title{
Optical precursors in the singular and weak dispersion limits
}

\author{
Kurt E. Oughstun, ${ }^{1, *}$ Natalie A. Cartwright, ${ }^{2}$ Daniel J. Gauthier, ${ }^{3}$ and Heejeong Jeong ${ }^{4}$ \\ ${ }^{1}$ College of Engineering and Mathematical Sciences, University of Vermont, Burlington, Vermont 05405, USA \\ ${ }^{2}$ Department of Mathematics, State University of New York at New Paltz, New Paltz, New York 12561, USA \\ ${ }^{3}$ Department of Physics, Duke University, Durham, North Carolina 27708, USA \\ ${ }^{4}$ Thayer School of Engineering, Dartmouth College, Hanover, New Hampshire 03755, USA \\ *Corresponding author: oughstun@cems.uvm.edu
}

Received May 4, 2010; revised June 22, 2010; accepted June 23, 2010;

posted June 23, 2010 (Doc. ID 127939); published July 29, 2010

\begin{abstract}
The description of the precursor fields in a single-resonance Lorentz model dielectric is considered in the singular and weak dispersion limits. The singular dispersion limit is obtained as the damping approaches zero and the material dispersion becomes increasingly concentrated about the resonance frequency. The algebraic peak amplitude decay of the Brillouin precursor with propagation distance $z>0$ then changes from a $z^{-1 / 2}$ to a $z^{-1 / 3}$ behavior. The weak dispersion limit is obtained as the material density decreases to zero. The material dispersion then becomes vanishingly small everywhere and the precursors become increasingly compressed in the space-time domain immediately following the speed-of-light point $(z, t)=(z, z / c)$. In order to circumvent the numerical difficulties introduced in this case, an approximate equivalence relation is derived that allows the propagated field evolution due to an ultrawideband signal to be calculated in an equivalent dispersive medium that is highly absorptive. (C) 2010 Optical Society of America
\end{abstract}

OCIS codes: $260.2030,320.5550,320.2250$.

\section{INTRODUCTION}

The asymptotic theory of ultrawideband dispersive pulse propagation in a Lorentz model dielectric [1] has its origin in the now classic research by Sommerfeld [2] and Brillouin $[3,4]$, which established the physical phenomena of the forerunners, or precursor fields as they were later named by Stratton [5], which are associated with the space-time evolution of a Heaviside step function signal. This asymptotic description is derived from the exact Fourier-Laplace integral representation of the propagated plane wave field [4-8], given by

$$
A(z, t)=\frac{1}{2 \pi} \int_{C} \tilde{f}(\omega) e^{(z / c) \phi(\omega, \theta)} d \omega,
$$

for all $z \geq 0$. Here the contour $C$ is the straight line path $\omega=\omega^{\prime}+i a$ with $\omega^{\prime}$ varying from negative to positive infinity along the real axis and with the constant $a$ chosen larger than the abscissa of absolute convergence [5-8] for the initial pulse spectrum,

$$
\widetilde{f}(\omega)=\int_{-\infty}^{\infty} f(t) e^{i \omega t} d t,
$$

of the initial plane wave pulse $A(0, t)=f(t)$ at $z=0$ inside the dispersive medium. Here $A(z, t)$ represents any scalar component of the plane wave electric or magnetic field vector whose temporal Fourier spectrum $\widetilde{A}(z, \omega)$ satisfies the Helmholtz equation

$$
\left(\nabla^{2}+\widetilde{k}^{2}(\omega)\right) \tilde{A}(z, \omega)=0
$$

with complex wave number $\tilde{k}(\omega)=\beta(\omega)+i \alpha(\omega)$ given by

$$
\widetilde{k}(\omega) \equiv \frac{\omega}{c} n(\omega)
$$

in the temporally dispersive medium with complex index of refraction $n(\omega)=n_{r}(\omega)+i n_{i}(\omega)$ whose real $n_{r}(\omega)$ $\equiv \mathfrak{R}\{n(\omega)\}$ and imaginary $n_{i}(\omega) \equiv \mathfrak{J}\{n(\omega)\}$ parts are related through the Kramers-Kronig relations [7,9]. This is an essential feature of any linear dispersive system so that due care must be taken in its proper physical description in any dispersive-wave propagation problem, especially in the ultrashort pulse an ultrawideband signal regime. Here $\beta(\omega) \equiv \mathfrak{R}\{\tilde{k}(\omega)\}$ is the propagation (or phase) factor and $\alpha(\omega) \equiv \mathfrak{J}\{\widetilde{k}(\omega)\}$ is the attenuation factor for plane wave propagation in the dispersive attenuative medium. In addition,

$$
\phi(\omega, \theta) \equiv \underset{z}{i-[\widetilde{k}}(\omega) z-\omega t]=i \omega[n(\omega)-\theta]
$$

is the complex phase function, where

$$
\theta \equiv \frac{c t}{z}, z>0
$$

is a nondimensional space-time parameter. Based on this integral representation, the modern asymptotic theory $[6,8,10-12]$ has provided a complete uniform asymptotic description of dispersive pulse dynamics for a variety of canonical pulse shapes and signals in a single-resonance Lorentz model dielectric with a causal complex index of refraction given by $[1,7,9]$ 


$$
n(\omega)=\left(1-\frac{\omega_{p}^{2}}{\omega^{2}-\omega_{0}^{2}+2 i \delta \omega}\right)^{1 / 2}
$$

Here $\omega_{0}$ is the resonance frequency, $\delta$ is the phenomenological damping constant, and $\omega_{p}^{2}=4 \pi N q_{e}^{2} / m_{e}$ is the square of the plasma frequency with number density $N$ $\geq 0$ of Lorentz oscillators in the medium, with $q_{e}$ denoting the charge magnitude and $m_{e}$ denoting the mass of the bound electron. The absorption band, which corresponds to the region of anomalous dispersion of the dielectric medium, then approximately extends from $\sqrt{\omega_{0}^{2}-\delta^{2}}$ to $\sqrt{\omega_{1}^{2}-\delta^{2}}$ along the positive real frequency axis, where $\omega_{1}$ $\equiv \sqrt{\omega_{0}^{2}+\omega_{p}^{2}}$. Because Eq. (7) is a causal model of the material dispersion, Sommerfeld's relativistic causality theorem holds $[2,6,8,10]$, which states that if $A(0, t)=0$ for all $t<0$, then $A(z, t)=0$ for all $\theta<1$ with $z>0$.

For $\theta \geq 1$, the asymptotic theory [3-6,8,10-12] has shown that the propagated wave field due to an ultrawideband signal [13] in a single-resonance Lorentz model dielectric may be expressed either as the linear superposition of three component fields as

$$
A(z, t)=A_{S}(z, t)+A_{B}(z, t)+A_{c}(z, t),
$$

as $z \rightarrow \infty$, as for the Heaviside unit step function signal $f(t)=u_{H}(t) \sin \left(\omega_{c} t\right)$, where $u_{H}(t<0)=0$ and $u_{H}(t>0)=1$, or as a linear combination of fields of this form, as for a rectangular envelope pulse. Here $A_{S}(z, t)$ is the first forerunner [2-4] or Sommerfeld precursor describing the highfrequency $\left(|\omega| \geq \omega_{1}\right)$ response of the dispersive medium, $A_{B}(z, t)$ is the second forerunner [3,4] or Brillouin precursor describing the low-frequency $\left(|\omega| \leq \omega_{0}\right)$ response of the dispersive medium, and $A_{c}(z, t)$ is the pole contribution describing the signal contribution (if any). The observed pulse distortion for the Heaviside step function signal is then seen to be primarily due to interference between the signal contribution and the precursor fields. Of particular importance here is the fact that the peak amplitude point of the Brillouin precursor experiences zero exponential decay with propagation distance $z>0$, decreasing algebraically as $z^{-1 / 2}$ in the dispersive absorptive medium, with the peak values of both the energy density and Poynting vector then only decreasing algebraically as $z^{-1}$, thereby transmitting electromagnetic energy deeper into the dispersive attenuative dielectric $[8,14]$ than that described by the Beer-Lambert-Bouger law [15]. This rather unique feature renders the Brillouin precursor as a powerful tool for imaging through a given obscuring dispersive material $[8,16]$, a result that has been verified experimentally for large bandwidth microwave signals [17].

The material absorption in a Lorentz medium, as described by the amplitude attenuation coefficient $\alpha(\omega)$ $=(\omega / c) n_{i}(\omega)$ for real $\omega$ [see Eq. (4)], decreases either when $\delta \rightarrow 0$ or when $N \rightarrow 0$. In the first case, the material dispersion becomes increasingly localized about the resonance frequency as $\delta \rightarrow 0$ and so is referred to here as the singular dispersion limit. The term "singular" is used here in the mathematical sense that the function $\alpha(\omega)$ fails to be well-behaved at a point in some well-defined manner, in this case in terms of its differentiability at $\omega=\omega_{0}$. In the second case, the absorption vanishes while the material dispersion $n_{r}(\omega)$ approaches unity at all frequencies as
$N \rightarrow 0$ and so is referred to here as the weak dispersion limit. In this limit, $d n(\omega) / d \omega \rightarrow 0$ as $N \rightarrow 0$, in agreement with the group velocity interpretation of weak material dispersion [18].

The singular and weak dispersion limits have received considerable attention recently because, unlike in the microwave domain, it is easier to experimentally observe optical precursors when both limits hold. In that case, the material dispersion is accurately described by the Lorentz model. In particular Jeong et al. [19] observed precursorlike waveforms when a Heaviside step function signal propagated through a gas of cold potassium atoms. They found that the precursors have a large amplitude that is comparable to the amplitude of the incident signal and persist for many nanoseconds for the case when the signal frequency is very close to the resonance frequency of the Lorentz model dielectric $\left(\left|\omega_{c}-\omega_{0}\right| \lesssim \delta\right)$, making them easier to detect directly using standard fast detectors and oscilloscopes. This research prompted other studies [20], including the generation of optical precursors in a material displaying electromagnetically induced transparency [21,22]; the interplay between optical precursors, selfinduced transparency [23], and free-induction decay [24]; and the so-called precursor stacking [25].

In these recent studies [20-24], the theoretical analysis proceeds by evaluating the integral in Eq. (1) using an approximate simplified expression for the complex index of refraction. With such an approximation, it is possible to obtain either approximate analytical expressions for the Sommerfeld $A_{S}(z, t)$ and Brillouin $A_{B}(z, t)$ precursors, as well as for the signal contribution $A_{c}(z, t)$, or hybrid numeric-analytic solutions. Unlike the uniform asymptotic description $[8,12]$ which is valid over the entire subluminal space-time domain $\theta \geq 1$ of interest, no systematic analysis has been presented to place bounds on the accuracy of these approximate solutions. LeFew et al. [26], on the other hand, have determined the range of material and pulse parameters over which their approximate asymptotic description is valid. Specifically, they provide an approximate description of the propagated coherent optical field in this weak and singular dispersion limit that is valid when the resonance frequency $\omega_{0}$ is much larger than both the plasma frequency $\omega_{p}$ and the damping $\delta$. This then restricts the space-time domain over which this description is valid to some subluminal subdomain $\left(\theta_{\min }, \theta_{\max }\right)$ of the space-time parameter $\theta$ with $\theta_{\max }>\theta_{\min }>\theta_{0}$, where

$$
\theta_{0} \equiv n(0)=\sqrt{1+\omega_{p}^{2} / \omega_{0}^{2}}
$$

with their analysis being designed to describe the experimental results of Jeong et al. [19]. In order to obtain this approximate description, they expand the complex phase function $\phi(\omega, \theta)$ about the medium resonance frequency $\omega_{0}$, neglect higher-order terms, and determine the saddle points of the resultant approximate phase function. Their results reduce to those of Crisp [27] when the local field effects may be considered negligible. Specifically, the parameters of validity given in Eqs. (60) and (61) by LeFew et al. [26] result in the inequality 


$$
\left(\omega_{p} / \omega_{0}\right)^{2} \ll \theta-1 \ll\left(\omega_{0} / \omega_{p}\right)^{2},
$$

and the inequalities given in Eqs. (59) and (62) of their paper [26] result in the inequality

$$
\left(\frac{2 c}{\omega_{p} z}\right)^{2} \ll \theta-1 \ll\left(\frac{\omega_{0} c}{\omega_{p} \delta z}\right)^{2} .
$$

Most revealing is the inequality given in Eq. (10) above. If one wants to describe the dominant behavior in the precursor fields, which occurs soon after the pulse front at $\theta=1$ in a weakly dispersive medium, one needs to evaluate Eq. (1) about the space-time point $\theta=\theta_{0}$ given in Eq. (9). Substitution of this value into Eq. (10) then yields the inequality $0 \gg\left(\omega_{p} / \omega_{0}\right)^{2}+\left(\omega_{p} / \omega_{0}\right)^{4}$, which is impossible to satisfy. Hence, the method of LeFew et al. [26], as well as that of Crisp [27], can only describe the late space-time behavior of the precursor fields, in agreement with the comment by Macke and Ségard [28]. Therein lies part of the purpose of the analysis presented in this paper.

These two limiting cases of singular and weak dispersion are fundamentally different in their effects upon ultrawideband pulse propagation and are thus treated separately in the following two sections, with the approach presented here for the weak dispersion case providing a complete description of the entire precursor evolution in contrast to the partial late-time description of the Brillouin precursor evolution that is provided by the approximate description of LeFew et al. [26].

\section{ULTRAWIDEBAND PULSE DYNAMICS IN THE SINGULAR DISPERSION LIMIT}

The asymptotic theory $[6,8,10]$ shows that the first-order near saddle points $S P_{n}^{ \pm}$of the complex phase function $\phi(\omega, \theta)$ for a single-resonance Lorentz medium coalesce into a single second-order saddle point $S P_{n}$ at

$$
\omega_{S P_{n}^{ \pm}}\left(\theta_{1}\right) \simeq-\frac{2 \delta}{3 \alpha_{c f}} i,
$$

when $\theta=\theta_{1}$, where $[6,8,10]$

$$
\theta_{1} \simeq \theta_{0}+\frac{2 \delta^{2} \omega_{p}^{2}}{3 \alpha_{c f} \theta_{0} \omega_{0}^{4}},
$$

with $\alpha_{c f} \simeq 1$ (a more accurate expression for this correction factor is given in $[6,8,10])$. At the space-time point $\theta=\theta_{0}$, the dominant near saddle point $S P_{n}^{+}$crosses the origin $\left[\omega_{S P_{n}^{+}}\left(\theta_{0}\right)=0\right]$ so that its contribution to the asymptotic behavior of the propagated wave field experiences zero exponential attenuation, viz.,

$$
\phi\left(\omega_{S P_{n}^{+}}\left(\theta_{0}\right), \theta_{0}\right)=0,
$$

with the peak amplitude point in the wave field decaying only as $z^{-1 / 2}$ with $z \rightarrow \infty$. At the subsequent space-time point $\theta=\theta_{1}$, this contribution to the asymptotic wave experiences a small (but nonzero) amount of exponential attenuation with the propagation distance as well as a $z^{-1 / 3}$ algebraic decay as $z \rightarrow \infty$, provided that $\delta>0$. In the singular dispersion limit as $\delta \rightarrow 0$, however, the two near saddle points $S P_{n}^{ \pm}$coalesce into a single second-order saddle point at the origin when $\theta=\theta_{1} \rightarrow \theta_{0}$, resulting in a peak amplitude that experiences zero exponential attenuation, with the amplitude now decaying only as $z^{-1 / 3}$ as $z \rightarrow \infty$. An estimate of the space-time interval $\left(\theta_{0}-\Delta \theta, \theta_{0}\right.$ $+\Delta \theta)$ about $\theta_{0}$ during which the Brillouin precursor amplitude reaches its peak amplitude is provided by $\Delta \theta$ $\approx\left(2 \delta \omega_{p}\right)^{2} /\left(3 \theta_{0} \omega_{0}^{4}\right)$, resulting in

$$
T_{\mathrm{eff}} \approx \frac{8 \delta^{2} \omega_{p}^{2} z}{3 \theta_{0} \omega_{0}^{4} c},
$$

as an estimate of its effective temporal width as $z \rightarrow \infty$. This value describes the half-period of the Brillouin precursor about $\theta=\theta_{0}$ so that $1 /\left(2 T_{\text {eff }}\right)$ describes its effective oscillation frequency (in hertz) at that critical space-time point as $z \rightarrow \infty$ (see Section 13.3.3 of [8] as well as Figs. 6 and 7 of [16]).

The numerically determined peak amplitude decay with relative propagation distance $z / z_{d}$ is presented in Fig. 1 for an input Heaviside unit step function modulated signal

$$
A(0, t)=f(t)=u_{H}(t) \sin \left(\omega_{c} t\right),
$$

with a below resonance angular carrier frequency $\omega_{c}$ $=3.0 \times 10^{14} \mathrm{rad} / \mathrm{s}$ in a single-resonance Lorentz model dielectric with a resonance frequency $\omega_{0}=3.9 \times 10^{14} \mathrm{rad} / \mathrm{s}$ (chosen to correspond to the experimental value in [19]) and a plasma frequency $\omega_{p}=3.05 \times 10^{14} \mathrm{rad} / \mathrm{s}$ for several values of the damping constant $\delta$. Here

$$
z_{d} \equiv \alpha^{-1}\left(\omega_{c}\right)
$$

denotes the $e^{-1}$ amplitude penetration depth at $\omega_{c}$. Notice that $z_{d}$ also depends on the damping constant $\delta$ through Eqs. (4) and (7). Nevertheless, its value is constant for each curve in Figs. 1 and 2 , varying from $z_{d} \approx 7.69 \mu \mathrm{m}$ when $\delta=\delta_{0}=3.02 \times 10^{13} \mathrm{rad} / \mathrm{s}$ to $z_{d} \approx 0.72 \mathrm{~cm}$ when $\delta$ $=0.001 \delta_{0}$. The dashed line in Fig. 1 describes the pure exponential attenuation described by the Beer-LambertBouger law relation $e^{-z / z}$ for the amplitude decay. The peak amplitude value used here is given by the measured

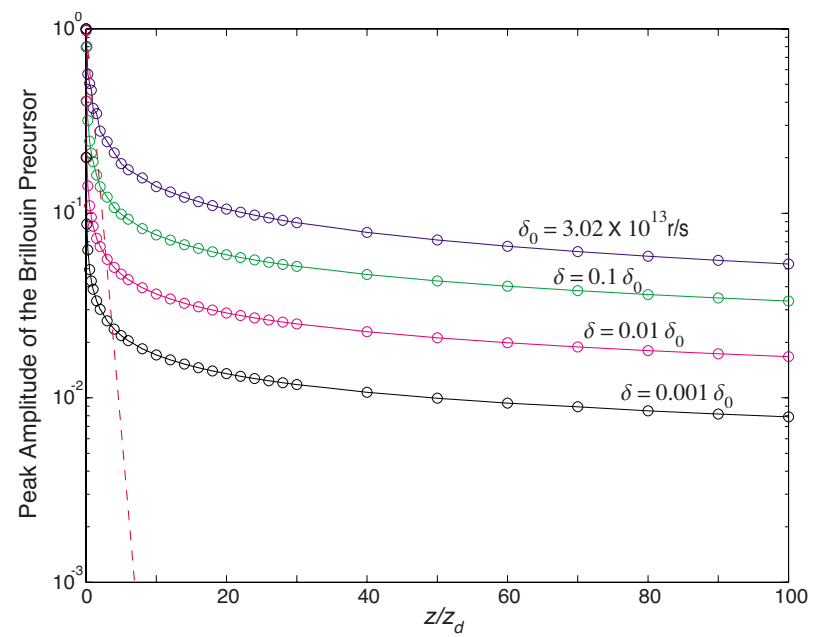

Fig. 1. (Color online) Numerically determined peak amplitude decay of a Heaviside unit step function signal with below resonance carrier frequency $\omega_{c} \simeq 0.769 \omega_{0}$ in a single-resonance Lorentz model dielectric as a function of the relative propagation distance $z / z_{d}$ for several decreasing values of the phenomenological damping constant $\delta>0$. 


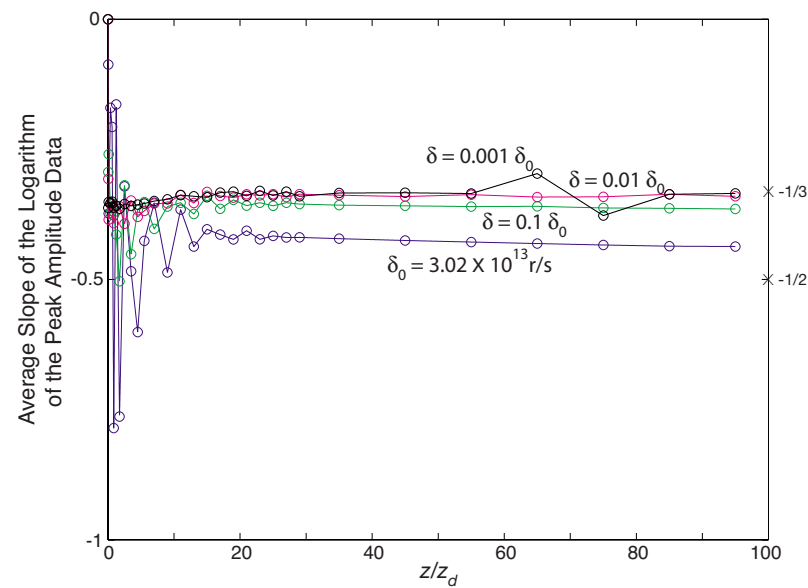

Fig. 2. (Color online) Average slope of the base ten logarithm of the numerical data presented in Fig. 1. The discontinuous behavior exhibited in the $\delta_{0} / 1000$ case is attributed to numerical error, which may be eliminated by increasing the number of sample points used to calculate the wave field.

amplitude of the first maximum appearing in the temporal evolution of the numerically determined propagated pulse at a fixed observation distance $z \geq 0$. Notice that this "leading-edge" peak amplitude point initially attenuates more rapidly than that of the signal oscillating at $\omega$ $=\omega_{c}$, but that as the mature dispersion regime is reached and the Brillouin precursor structure emerges from the propagated wave field, a transition is made from exponential attenuation to algebraic decay. Notice further that this transition occurs at a larger relative propagation distance $z / z_{d}$ as $\delta$ decreases and the medium dispersion becomes increasingly localized about $\omega_{0}$ and, hence, more singular.

The algebraic power of the measured peak amplitude attenuation presented in Fig. 1 may be determined [16] by plotting the logarithm of the peak amplitude data $A_{\text {peak }}=B\left(z / z_{d}\right)^{p}$ versus the logarithm of the relative propagation distance $z / z_{d}$. The numerically determined average slope of the base ten logarithm of the data present in Fig. 1 is given in Fig. 2 for each value of $\delta$ considered. These numerical results show that the power $p$ increases from a value approaching $-1 / 2$ as $z / z_{d} \rightarrow \infty$ to a value approaching $-1 / 3$ as $z / z_{d} \rightarrow \infty$ when $\delta$ is decreased such that the inequality $\delta / \omega_{0} \ll 1$ is satisfied.

As the material dispersion becomes increasingly singular, the number of sample points required to accurately model the material dispersion and resultant propagated wave field structure increases. At the smallest value of $\delta$ considered here, the $2^{23}$ point fast Fourier transform (FFT) used was at the sampling limit with $\omega_{\max }=2 \pi$ $\times 10^{16} \mathrm{rad} / \mathrm{s}$, resulting in the small numerical error illustrated in Fig. 2. In this "extreme" case, a small change in the numerical sampling frequency by decreasing $\omega_{\max }$ by $1 / 2$ results in small changes in the third significant figure of the numerically determined peak amplitude values. Although this numerical error is not evident in the peak amplitude plot in Fig. 1, it does result in a noticeable change in the numerically determined slope data presented in Fig. 2. This numerical error can be eliminated by increasing the sampling size of the FFT algorithm beyond the $2^{23}$ point limit of the computer system used in this study.

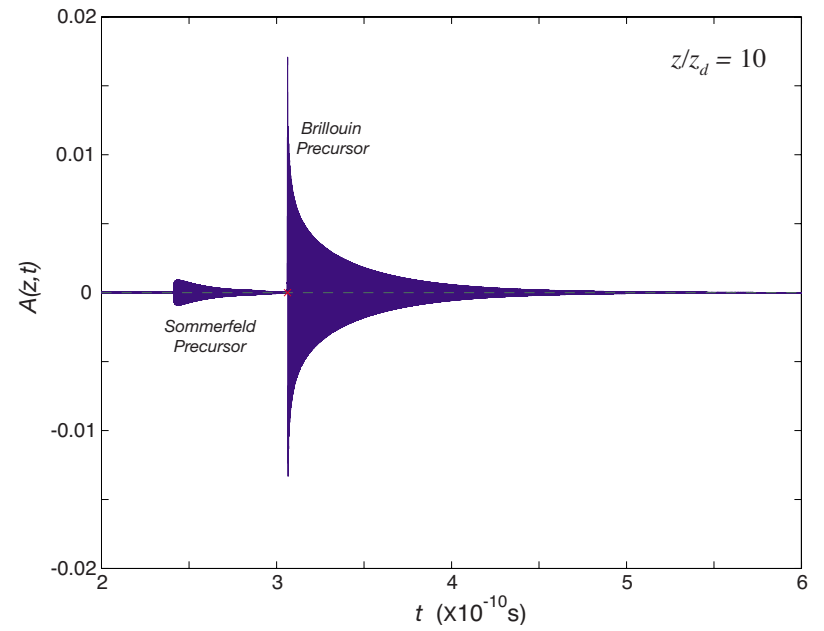

Fig. 3. (Color online) Propagated plane wave field at ten absorption depths due to an input Heaviside unit step function modulated signal starting at time $t=0$ at the plane $z=0$ with below resonance angular carrier frequency $\omega_{c} \simeq 0.769 \omega_{0}$ in a singleresonance Lorentz model dielectric. The cross-symbol in the plot marks the time $t_{0}=\theta_{0} z / c$ following the onset of the Brillouin precursor.

An example of the numerically computed dynamical wave field evolution in the singular dispersion limit is presented in Fig. 3. The initial wave field at $z=0$ is the Heaviside unit step function signal given in Eq. (14) with a below resonance angular carrier frequency of $\omega_{c}=3.0$ $\times 10^{14} \mathrm{rad} / \mathrm{s}$. The propagated wave field was calculated at ten absorption depths into a single-resonance Lorentz medium with resonance frequency $\omega_{0}=3.9 \times 10^{14} \mathrm{rad} / \mathrm{s}$, plasma frequency $\omega_{p}=3.05 \times 10^{14} \mathrm{rad} / \mathrm{s}$, and phenomenological damping constant $\delta=3.02 \times 10^{10} \mathrm{rad} / \mathrm{s}$. Because $\delta / \omega_{0}=7.74 \times 10^{-5}$, this case is well within the singular dispersion regime. A distinguishing feature of this field evolution in the singular dispersion limit is the sharp leading edge of the Brillouin precursor just preceding the spacetime point $c t / z=\theta_{0}$ at which the peak amplitude point occurs, followed by a relatively slow decay in the field amplitude as time $t$ increases above $\theta_{0} z / c$ with $z>0$. The rate of this decay with the propagation distance for $\theta$ $>\theta_{0}$ is determined by the absorption coefficient $\alpha(\omega)$ $\equiv \mathfrak{J}\{\tilde{k}(\omega)\}$ evaluated at the near saddle point $S P_{n}^{+}$, which is found to be proportional to the damping constant $\delta$ (see Eq. (12.240) of [8]).

\section{ULTRAWIDEBAND PULSE DYNAMICS IN THE WEAK DISPERSION LIMIT}

In the weak dispersion limit as $N \rightarrow 0$, the material dispersion approaches that for vacuum at all frequencies, i.e., $n(\omega) \rightarrow 1$. This then introduces a rather curious difficulty into the numerical FFT simulation of pulse propagation in this weak dispersion limit as the number of sample points required to accurately model the propagated pulse behavior rapidly increases as the number density $N$ goes to zero. In order to circumvent this problem, we develop an approximate equivalence relation that allows us to compute the propagated wave field behavior in an equivalent dispersive medium that is strongly dispersive. This approximate equivalence relation, which be- 
comes exact in the limit as $N \rightarrow 0$ for each given medium, follows from the integral representation of the propagated wave field given in Eq. (1).

Two different propagation problems for the same initial pulse $A(0, t)=f(t)$ are identical provided that the relation

$$
\widetilde{k}_{1}(\omega) z_{1}-\omega t_{1}=\widetilde{k}_{2}(\omega) z_{2}-\omega t_{2}
$$

is satisfied for all $\omega$. Equating real and imaginary parts for real $\omega$ results in the pair of relations

$$
\begin{gathered}
\beta_{1}(\omega) z_{1}-\omega t_{1}=\beta_{2}(\omega) z_{2}-\omega t_{2}, \\
\alpha_{1}(\omega) z_{1}=\alpha_{2}(\omega) z_{2}
\end{gathered}
$$

both of which must be satisfied for all $\omega$. For the absorptive part, the equivalence relation

$$
z_{2}=\frac{\alpha_{1}(\omega)}{\alpha_{2}(\omega)} z_{1}, \quad \forall \omega
$$

results. If the two media differ only through their densities, then

$$
n(\omega)=\sqrt{1+N g(\omega)} \rightarrow 1+\frac{1}{2} N g(\omega) \quad \text { as } N \rightarrow 0,
$$

because $\alpha(\omega)=(\omega / c) n_{i}(\omega)$ for real $\omega$, where $g(\omega)$ $=-\left(4 \pi q_{e}^{2} / m_{e}\right) /\left(\omega^{2}-\omega_{0}^{2}+2 i \delta \omega\right)$. In this case, $n_{i}(\omega)$ $\approx \frac{1}{2} N g_{i}(\omega)$ and the above equivalence relation becomes

$$
z_{2} \approx \frac{N_{1}}{N_{2}} z_{1} \text {. }
$$

The accuracy of this result increases as $N_{1}, N_{2} \rightarrow 0$ with $N_{2}>N_{1}$, assuming that $z_{1}>z_{2}$. The corresponding equivalence relation for the phase part then becomes

$$
\begin{aligned}
\frac{\omega}{c}\left(1+\frac{1}{2} N_{1} g_{r}(\omega)\right) z_{1}-\omega t_{1} & \approx \frac{\omega}{c}\left(1+\frac{1}{2} N_{2} g_{r}(\omega)\right) z_{2}-\omega t_{2} \\
& \approx \frac{\omega}{c}\left(1+\frac{1}{2} N_{2} g_{r}(\omega)\right) \frac{N_{1}}{N_{2}} z_{1}-\omega t_{2}
\end{aligned}
$$

so that

$$
t_{2} \approx t_{1}+\left(\frac{N_{1}}{N_{2}}-1\right) \frac{z_{1}}{c},
$$

which is the second part of the desired equivalence relation. Because $\omega_{p}=\sqrt{4 \pi N q_{e}^{2} / m_{e}}$, the pair of equivalence relations given in Eqs. (22) and (23) may be expressed as

$$
\begin{gathered}
z_{2} \approx \frac{\omega_{p 1}^{2}}{\omega_{p 2}^{2}} z_{1}, \\
t_{2} \approx t_{1}+\left(\frac{\omega_{p 1}^{2}}{\omega_{p 2}^{2}}-1\right) \frac{z_{1}}{c} .
\end{gathered}
$$

Notice that the second relation is simply a uniform displacement in time. The first part of this equivalence relation is the essential part. For example, if $N_{1} / N_{2}=1$ $\times 10^{-2}$, then $z_{2}=z_{1} \times 10^{-2}$ and $t_{2}=t_{1}-\left(0.33 \times 10^{-8} \mathrm{~s} / \mathrm{m}\right) z_{1}$. In that case, the propagated wave field structure illus- trated in Fig. 3 also applies to the case when the medium plasma frequency $\omega_{p}$ is reduced by a factor of 10 and the propagation distance $z$ is increased by a factor of 100 provided that the time origin is adjusted according to Eq. (23).

The accuracy of this equivalence relation is illustrated in the sequence of wave field plots presented in Figs. 4-6. The initial pulse in each case is the Heaviside unit step function modulated signal given in Eq. (16) with below resonance angular carrier frequency $\omega_{c}=3.0 \times 10^{14} \mathrm{rad} / \mathrm{s}$ in a single-resonance Lorentz model dielectric with resonance frequency $\omega_{0}=3.90 \times 10^{14} \mathrm{rad} / \mathrm{s}$ and phenomenological damping constant $\delta=3.02 \times 10^{12} \mathrm{rad} / \mathrm{s}$. The plasma frequency in case 1 illustrated in Fig. 4 is $\omega_{p 1}$ $=3.05 \times 10^{14} \mathrm{rad} / \mathrm{s}$, resulting in an absorption depth of $z_{d} \equiv \alpha^{-1}\left(\omega_{c}\right)=7.238 \times 10^{-5} \mathrm{~m}$. In case 2, illustrated in Fig. 5 , the plasma frequency has been reduced by 2 orders of magnitude (and the number density by 4 orders of magnitude) to the value $\omega_{p 2}=3.05 \times 10^{12} \mathrm{rad} / \mathrm{s}$, resulting in an absorption depth of $z_{d} \equiv \alpha^{-1}\left(\omega_{c}\right)=0.458 \mathrm{~m}$. Finally, in case 3, illustrated in Fig. 6, the plasma frequency has been reduced by an additional 2 orders of magnitude (and the number density by 8 orders of magnitude from that in case 1) to the value $\omega_{p 3}=3.05 \times 10^{10} \mathrm{rad} / \mathrm{s}$, resulting in an absorption depth of $z_{d} \equiv \alpha^{-1}\left(\omega_{c}\right)=4.582 \times 10^{3} \mathrm{~m}$. Each of these propagated wave field structures was computed at $z=5 z_{d}$ with a $2^{23}$ point $\mathrm{FFT}$ and the time origin in each was shifted by the amount $z / c$ in order to align them temporally for the sole purpose of ease of comparison.

Each of the propagated wave field structures presented in Fig. 4-6 exhibits the field structure described by the asymptotic theory given in Eq. (8) for a below resonance carrier frequency, with the high-frequency Sommerfeld precursor $A_{S}(z, t)$ arriving at the speed-of-light point $t$ $=z / c$, followed by the evolution of the low-frequency Brillouin precursor $A_{B}(z, t)$, which is then followed by the signal contribution $A_{c}(z, t)$ that is primarily oscillating at the angular carrier frequency $\omega_{c}$ of the input signal $[6,8,10]$. Notice that each wave field evolution was calculated at five absorption depths $\left(z=5 z_{d}\right)$ at the carrier frequency $\omega_{c}$, which translates into applying the equivalence relation in

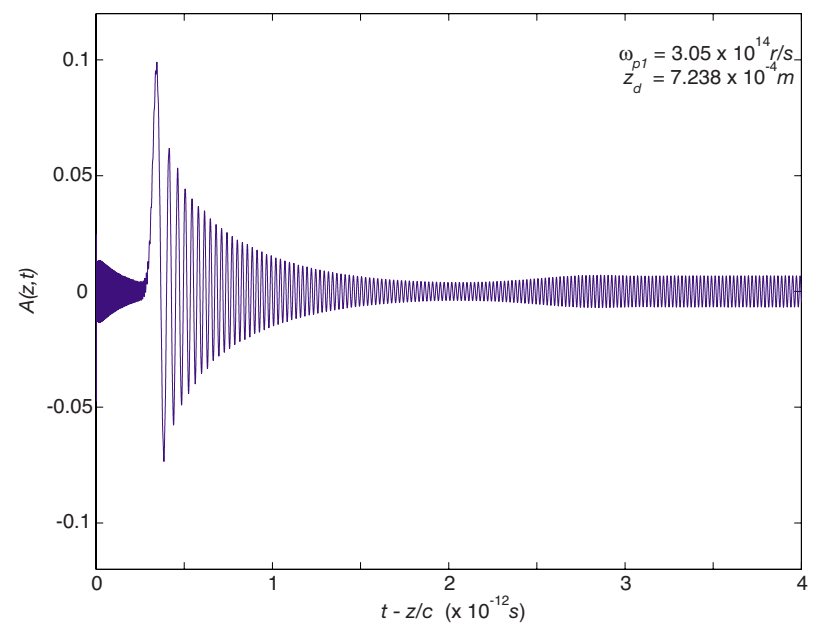

Fig. 4. (Color online) Propagated wave field at five absorption depths in a single-resonance Lorentz medium with plasma frequency $\omega_{p 1}=3.05 \times 10^{14} \mathrm{rad} / \mathrm{s}$. Notice that $A(z, t)=0$ for all $t<z / c$. 


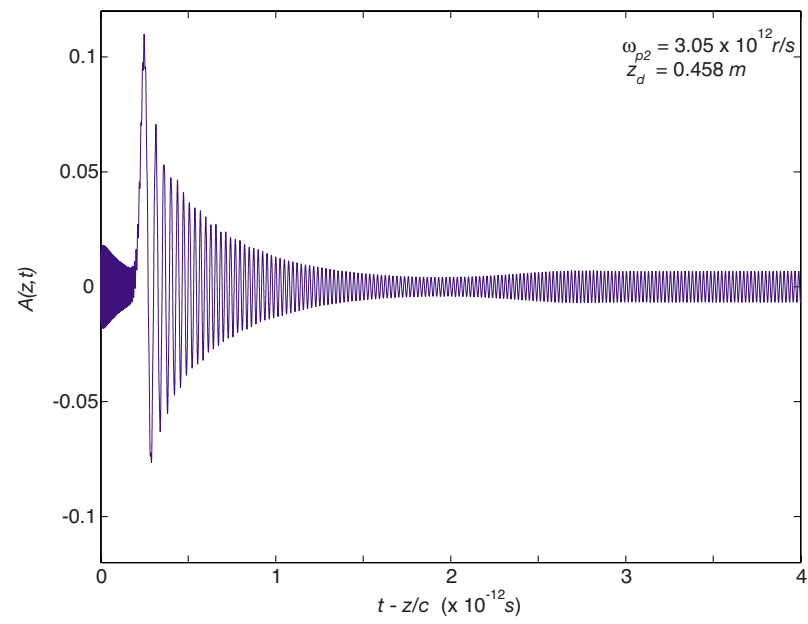

Fig. 5. (Color online) Propagated wave field at five absorption depths in a single-resonance Lorentz medium with plasma frequency $\omega_{p 2}=3.05 \times 10^{12} \mathrm{rad} / \mathrm{s}$. Notice that $A(z, t)=0$ for all $t<z / c$.

Eq. (19) at the carrier frequency alone. Although this is inaccurate when the number density $N$ is large, it becomes increasingly accurate as $N$ decreases to zero. Comparison of these field structure shows that the propagated field changes almost imperceptibly as one goes from the optically dense medium in case 1 (Fig. 4) to the optically rare medium in case 2 (Fig. 5) and that there is no discernible further change as one then progresses to the optically rarer media in case 3 (Fig. 6). The field evolution described in Figs. 5 and 6 will then remain valid, subject to the equivalence relations given in Eqs. (24) and (25), as the medium density is further decreased.

The unique advantage of the modern asymptotic description lies in the fact that the complete space-time evolution of each component of the propagated wave field is described separately from the other components. Each individual field component, as described by the uniform asymptotic theory [8,12] with numerically determined saddle point locations, is presented in Fig. 7 for case 2 (cf. Fig. 5). These results clearly reveal the full space-time

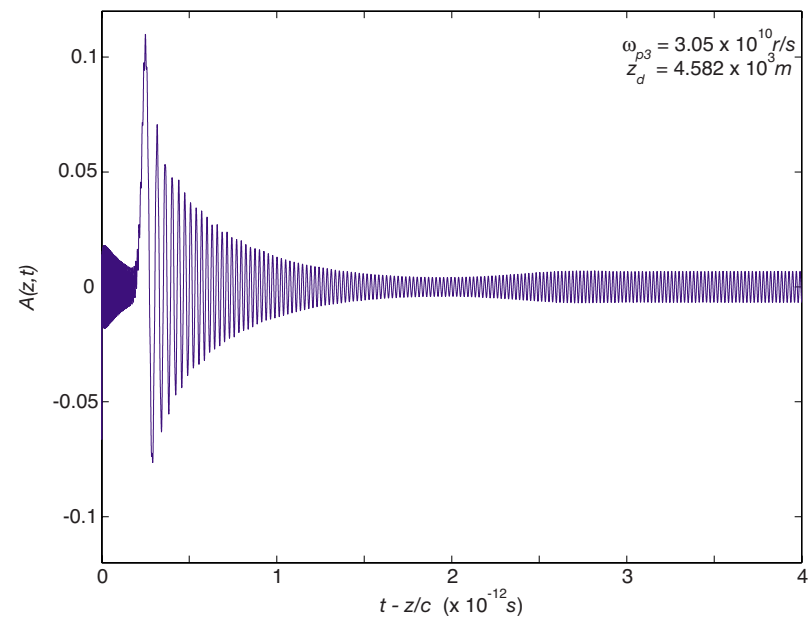

Fig. 6. (Color online) Propagated wave field at five absorption depths in a single-resonance Lorentz medium with plasma frequency $\omega_{p 3}=3.05 \times 10^{10} \mathrm{rad} / \mathrm{s}$. Notice that $A(z, t)=0$ for all $t<z / c$.

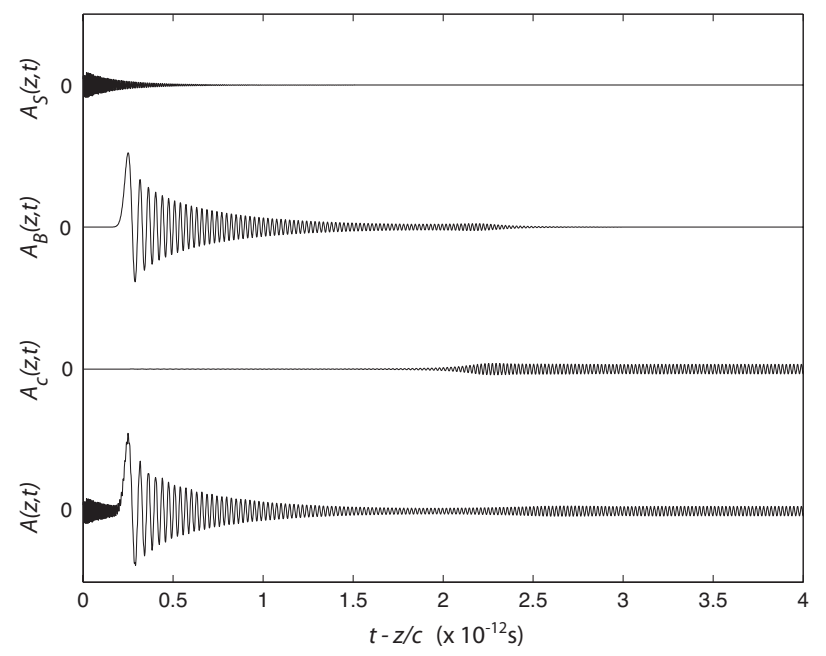

Fig. 7. Separate Sommerfeld precursor $A_{S}(z, t)$, Brillouin precursor $A_{B}(z, t)$, and signal $A_{c}(z, t)$ components of the propagated wave field and their superposition to yield the total propagated field $A(z, t)$ at five absorption depths in a single-resonance Lorentz medium with plasma frequency $\omega_{p 2}=3.05 \times 10^{12} \mathrm{rad} / \mathrm{s}$. Notice that $A(z, t)=0$ for all $t<z / c$.

evolution of the separate Sommerfeld $A_{S}(z, t)$ and Brillouin $A_{B}(z, t)$ precursors as well as the signal evolution $A_{c}(z, t)$ that is partially obscured in the full field evolution $A(z, t)$. Finally, notice again that the approximate equivalence relations given in Eqs. (22)-(25) become increasingly accurate as the number density is decreased, thereby extending this modern asymptotic description into the weak dispersion limit.

\section{SUMMARY}

The description of the precursor fields in a singleresonance Lorentz model dielectric has been presented for both the singular and weak dispersion limits. The singular dispersion limit is obtained as the damping approaches zero and the material dispersion becomes increasingly concentrated about the resonance frequency. The algebraic peak amplitude decay of the Brillouin precursor with the propagation distance $z>0$ was then shown to change from a $z^{-1 / 2}$ to a $z^{-1 / 3}$ behavior, a phenomenon described by the asymptotic theory of dispersive pulse propagation $[3,4,8]$. The weak dispersion limit is obtained as the material density decreases to zero. The material dispersion then becomes vanishingly small everywhere and the precursors become increasingly compressed in the $\theta=c t / z$ space-time domain immediately following the speed-of-light point $\theta=1$. In order to circumvent the numerical difficulties introduced in this case, an approximate equivalence relation was derived that allows the propagated field evolution to be calculated in an equivalent dispersive medium that is highly absorptive.

The results presented here are for a Heaviside step function signal which possesses an idealized instantaneous turn-on. As shown in [29], the results will remain valid for a noninstantaneous turn-on signal provided that the signal turn-on time $T_{r}$ is faster than the characteristic relaxation time $1 / \delta$ of the material dispersion. 
A special challenge is presented by the experimental conditions of [19], which exhibits both weak and singular dispersion limits. Specifically, the single-resonance Lorentz model of the $\mathrm{K}$ magneto-optical trap with resonance frequency $\omega_{0}=2.4 \times 10^{15} \mathrm{rad} / \mathrm{s}$ employed in their study has a plasma frequency $\omega_{p}=6 \pi \times 10^{9} \mathrm{rad} / \mathrm{s}$, making it weakly dispersive with $\omega_{p} / \omega_{0} \approx 7.85 \times 10^{-7}$, and a damping constant $\delta \approx 9.6 \pi \times 10^{6} \mathrm{rad} / \mathrm{s}$, making the dispersion nearly singular with $\delta / \omega_{0} \approx 1.26 \times 10^{-8}$. For numerical computations using a FFT synthesis of Eq. (1) with the first form of Eq. (5), the weakly dispersive property can be directly alleviated by employing the equivalence relation presented in Section 3. However, the singular dispersion property exhibited by this material requires sampling that is far beyond the $2^{23}$ point FFT used in this study. In addition, this combination of weak and singular dispersions presents numerical difficulties in the precise determination of the near saddle point locations, a problem that can be resolved either by a more precise numerical root-finding algorithm or by a more accurate analytical solution [8].

\section{ACKNOWLEDGMENTS}

The research presented in this paper has been supported, in part, by the U.S. Air Force Office of Scientific Research (AFOSR) through AFOSR grant numbers FA9550-08-10097 and FA9550-07-1-0112.

\section{REFERENCES AND NOTES}

1. H. A. Lorentz, Theory of Electrons (Teubner, 1906), Chap. IV.

2. A. Sommerfeld, "Über die fortpflanzung des lichtes in disperdierenden medien,” Ann. Phys. (Leipzig) 44, 177-202 (1914).

3. L. Brillouin, "Über die fortpflanzung des licht in disperdierenden medien," Ann. Phys. (Leipzig) 44, 203-240 (1914).

4. L. Brillouin, Wave Propagation and Group Velocity (Academic, 1960)

5. J. A. Stratton, Electromagnetic Theory (McGraw-Hill, 1941), Sec. 5.18.

6. K. E. Oughstun and G. C. Sherman, Electromagnetic Pulse Propagation in Causal Dielectrics (Springer-Verlag, 1994).

7. K. E. Oughstun, Electromagnetic \& Optical Pulse Propagation 1: Spectral Representations in Temporally Dispersive Media (Springer, 2006).

8. K. E. Oughstun, Electromagnetic \& Optical Pulse Propagation 2: Temporal Pulse Dynamics in Dispersive Attenuative Media (Springer, 2009).

9. H. M. Nussenzveig, Causality and Dispersion Relations (Academic, 1972), Chap. 1.

10. K. E. Oughstun and G. C. Sherman, "Propagation of electromagnetic pulses in a linear dispersive medium with absorption (the Lorentz medium)," J. Opt. Soc. Am. B 5, 817849 (1988).

11. K. E. Oughstun and G. C. Sherman, "Uniform asymptotic description of electromagnetic pulse propagation in a linear dispersive medium with absorption (the Lorentz medium)," J. Opt. Soc. Am. A 6, 1394-1420 (1989).

12. N. A. Cartwright and K. E. Oughstun, "Uniform asymptotics applied to ultrawideband pulse propagation," SIAM Rev. 49, 628-648 (2007).
13. There are several definitions of what an ultrawideband signal is; see, for example, Section 11.2.2 of [8]. We take here the simple physical definition to mean a temporal pulse whose frequency spectrum along the positive real frequency axis is nonzero as $\omega \rightarrow 0$ and which goes to zero as $1 / \omega$ or less as $\omega \rightarrow \infty$.

14. P. D. Smith and K. E. Oughstun, "Electromagnetic energy dissipation and propagation of an ultrawideband plane wave pulse in a causally dispersive dielectric," Radio Sci. 33, 1489-1504 (1998).

15. The Beer-Lambert-Bouger law was originally discovered by P. Bouger, Essai d'Optique sur la Gradation de la Lumiere (Claude Jombert, 1729) and subsequently cited by J. H. Lambert, Photometri (V. E. Klett, 1760); the result was then extended by A. Beer, Einleitung in die höhere Optik (Friedrich Viewig, 1853) to include the concentration of solutions in the expression of the absorption coefficient for the intensity of light.

16. K. E. Oughstun, "Dynamical evolution of the Brillouin precursor in Rocard-Powles-Debye model dielectrics," IEEE Trans. Antennas Propag. 53, 1582-1590 (2005).

17. M. Pieraccini, A. Bicci, D. Mecatti, G. Macaluso, and C. Atzeni, "Propagation of large bandwidth microwave signals in water," IEEE Trans. Antennas Propag. 57, 3612-3618 (2009).

18. In the group velocity description, the "strength" of the material dispersion is typically measured through the derivative $d n_{r}(\omega) / d \omega$ as that is what appears in the coefficients $\beta_{1} \equiv\left(n_{r}+\omega d n_{r} / d \omega\right) / c$ and $\beta_{2} \equiv\left(2 d n_{r} / d \omega+\omega d^{2} n_{r} / d \omega^{2}\right) / c$ in the Taylor series expansion of the real propagation factor $\beta(\omega) \equiv \mathfrak{R}\{\widetilde{k}(\omega)\}$ in a hypothetical "lossless" dispersive medium. It is directly found from Eq. (7) that this first derivative is directly proportional to the number density $N$ so that these coefficients can always be made as small as desired at any real $\omega$ simply by choosing $N$ sufficiently small.

19. H. Jeong, A. M. C. Dawes, and D. J. Gauthier, "Direct observation of optical precursors in a region of anomalous dispersion,” Phys. Rev. Lett. 96, 143901 (2006).

20. B. Macke and B. Ségard, "Optical precursors in transparent media," Phys. Rev. A 80, 011803 (2009).

21. H. Jeong and S. Du, "Two-way transparency in the lightmatter interaction: Optical precursors with electromagnetically induced transparency," Phys. Rev. A 79, 011802 (2009).

22. D. Wei, J. F. Chen, M. M. T. Loy, G. K. L. Wong, and S. Du, "Optical precursors with electromagnetically induced transparency in cold atoms," Phys. Rev. Lett. 103, 093602 (2009).

23. B. Macke and B. Ségard, "Optical precursors with selfinduced transparency," Phys. Rev. A 81, 015803 (2010).

24. J. F. Chen, S. Wang, D. Wei, M. M. T. Loy, G. K. L. Wong, and S. Du, "Optical coherent transients in cold atoms: from free induction decay to optical precursors," Phys. Rev. A 81, 033844 (2010)

25. H. Jeong and S. Du, "Slow-light-induced interference with stacked optical precursors for square input pulses," Opt. Lett. 35, 124-126 (2010).

26. W. R. LeFew, S. Venakides, and D. J. Gauthier, "Accurate description of optical precursors and their relation to weakfield coherent optical transients," Phys. Rev. A 79, 063842 (2009).

27. M. D. Crisp, "Propagation of small-area pulses of coherent light through a resonant medium," Phys. Rev. A 1, 16041611 (1970).

28. B. Macke and B. Ségard, "Comment on "Direct observation of optical precursors in a region of anomalous dispersion"," arXiv:physics/0605039.

29. K. E. Oughstun, "Noninstantaneous, finite rise-time effects on the precursor field formation in linear dispersive pulse propagation," J. Opt. Soc. Am. A 12, 1715-1729 (1995). 